\title{
Reflets
}

Revue d'intervention sociale et communautaire

\section{Militantisme au sein des groupes de défense des droits des personnes locataires : pratiques démocratiques et limites organisationnelles}

\section{Activism within tenants' rights groups: Democratic practices and organizational limits}

\section{Geneviève Breault}

Volume 23, numéro 2, automne 2017

Militantisme et changement social. Partie 2

URI : https://id.erudit.org/iderudit/1043306ar

DOI : https://doi.org/10.7202/1043306ar

Aller au sommaire du numéro

Éditeur(s)

Reflets, Revue d'intervention sociale et communautaire

ISSN

1203-4576 (imprimé)

1712-8498 (numérique)

Découvrir la revue

Citer cet article

Breault, G. (2017). Militantisme au sein des groupes de défense des droits des personnes locataires : pratiques démocratiques et limites organisationnelles. Reflets, 23(2), 181-204. https://doi.org/10.7202/1043306ar
Résumé de l'article

Cet article s'intéresse aux limites organisationnelles et aux pratiques démocratiques visant le développement des personnes mobilisées au sein de groupes communautaires montréalais de défense de droits des personnes locataires. Ces organismes sont, selon l'appellation de Mathieu et Mercier (1991), des groupes d'intérêts ayant pour champ d'action l'amélioration des conditions de vie des personnes locataires et se définissant et se structurant à partir des rapports problématiques que ces personnes entretiennent sur le plan structurel. Or, les entrevues menées avec sept travailleuses et travailleurs issus de ces groupes nous révèlent que la place accordée aux militantes et militants dans les espaces de délibération, ainsi que dans l'orientation des actions, alimente d'importants questionnements idéologiques et professionnels. Près de quarante ans après la création de ces premiers groupes, nous proposons un regard critique sur leurs modes d'appréhension de l'idéal démocratique dans un contexte de renouvellement des pratiques démocratiques.
Tous droits réservés @ $@$ Reflets, Revue d’intervention sociale et communautaire, 2017
Ce document est protégé par la loi sur le droit d'auteur. L'utilisation des services d'Érudit (y compris la reproduction) est assujettie à sa politique d'utilisation que vous pouvez consulter en ligne. 


\section{Militantisme au sein des groupes de défense des droits des personnes locataires : pratiques démocratiques et limites organisationnelles}

Geneviève Breault, Ph.D.

Université du Québec à Montréal

\section{Résumé}

Cet article s'intéresse aux limites organisationnelles et aux pratiques démocratiques visant le développement des personnes mobilisées au sein de groupes communautaires montréalais de défense de droits des personnes locataires. Ces organismes sont, selon l'appellation de Mathieu et Mercier (1991), des groupes d'intérêts ayant pour champ d'action l'amélioration des conditions de vie des personnes locataires et se définissant et se structurant à partir des rapports problématiques que ces personnes entretiennent sur le plan structurel. Or, les entrevues menées avec sept travailleuses et travailleurs issus de ces groupes nous révèlent que la place accordée aux militantes et militants dans les espaces de délibération, ainsi que dans l'orientation des actions, alimente d'importants questionnements idéologiques et professionnels. Près de quarante ans après la création de ces premiers groupes, nous proposons un regard critique sur leurs modes d'appréhension de l'idéal démocratique dans un contexte de renouvellement des pratiques démocratiques.

Mots clés : défense de droits, locataires, organisation communautaire, pratiques démocratiques, militantisme

\section{Activism within tenants' rights groups: democratic practices and organizational limits}

\section{Abstract}

This article looks at the organizational limits and democratic practices for the development of people mobilized within tenants' rights groups in Montréal. These organizations are, to use the term of Mathieu and Mercier (1991), interest groups with the scope of 
improving tenants' living conditions, defining and structuring themselves based on the structural problems tenants face. Interviews conducted with seven of their workers reveal worries based on a professional and ideological basis concerning members' deliberation and decisions issues. Forty years after the creation of the first tenants groups, we offer a critical look at their experience of the democratic ideal in a context of a renewal of democratic practices.

Key words: advocacy, tenants, organizational limits, democratic practices, community action, activism

\section{Introduction}

Regroupant des personnes et des collectivités qui subissent des dénis de leurs droits, les organismes de défense collective des droits (DCD) québécois mènent des luttes collectives et contribuent aux débats publics en agissant comme révélateurs et dénonciateurs de divers problèmes sociaux. En matière de logement, les organismes de défense de droits prennent l'appellation de " comité logement ». Ils se composent d'une équipe de travailleuses et travailleurs rémunérés et de membres impliqués de façon citoyenne

"Adoptant une perspective critique, ce texte poursuit d'abord et avant tout l'objectif de lever le voile de façon lucide sur les paradoxes avec lesquels les travailleuses et travailleurs doivent jongler au quotidien pour aménager des pratiques en accord avec leurs idéaux de lutte et de justice sociale. » et bénévole, les militantes et militants. Le présent article s'intéresse à la place qu'occupent ces militantes et militants en s'interrogeant sur les pratiques démocratiques qui caractérisent les organisations dont elles et ils font partie. Pour ce faire, nous abordons le rôle des travailleuses et travailleurs et leurs relations avec les membres, les modèles d'intervention privilégiés, ainsi que les structures organisationnelles et le fonctionnement de celles-ci. Adoptant une perspective critique, ce texte poursuit d'abord et avant tout l'objectif de lever le voile de façon lucide sur les paradoxes avec lesquels les travailleuses et travailleurs doivent jongler au quotidien pour aménager des pratiques en accord avec leurs idéaux de lutte et de justice sociale.

Plusieurs auteurs ont déjà fait le constat du déclin important de la dimension collective et de l'approche citoyenne au sein des organismes communautaires. On rapporte une accentuation de l'individualisation dans l'intervention, ce qui interfere avec le développement de modes d'action plus collectifs, dont l'éducation populaire, et 
la prise en charge démocratique des organismes. Ces tendances révéleraient l'existence d'un fossé important entre les cadres de référence et les pratiques réelles (René, 2009) puisque les groupes ne sont pas organisés ni ne travaillent comme avant. Tirés vers la professionnalisation sous la pression de l'État, ils seraient devenus des agents de démantèlement de la communauté (Deslauriers, 2014), ce qui occasionnerait un nouveau paradigme (Lamoureux, 2010). Les nouvelles pratiques de financement, soit les partenariats avec le secteur public, la philanthropie et l'introduction de modèles de gouvernance liés à la "nouvelle gestion publique ", poseraient des défis, car elles s'accompagneraient d'une dépolitisation des problématiques sociales sur lesquelles travaillent les organismes communautaires (Depelteau, 2013). Calquées sur les logiques de l'entreprise privée, ces pratiques administratives impliqueraient la mesure de résultats à l'aide d'indicateurs et la reddition de comptes et insisteraient sur des référents idéologiques axés sur l'individu, cela au détriment d'une compréhension globale et collective des problématiques vécues (Depelteau, Fortier et Hébert, 2013).

À l'instar de ces multiples constats, l'originalité de ce texte réside dans le fait qu'il permet d'avoir accès au point de vue des intervenantes et intervenants qui travaillent au sein des organismes de défense des droits de personnes locataires, alors que la plupart des écrits sur la question concernent les organismes famille et/ou de services sociaux. Il est pertinent que l'on s'attarde aux organismes de défense collective des droits, car ils portent des visées de changement social et de développement du pouvoir d'agir.

\section{Locataires en colère, locataires solidaires!}

À la recherche de solutions aux problèmes touchant leurs quartiers, les premiers groupes de défense de droits des personnes locataires québécois émergent à la fin des années 1960 sous la forme de comités de citoyens, puis de comités d'action politique (René et Panet-Raymond, 1984). Ces groupes parviennent à se doter d'infrastructures grâce à des programmes fédéraux de création d'emplois, dont les PIL et Canada au travail ${ }^{1}$ (Milot, 1979, p. 3), qui créent de toute pièce de nouvelles associations de locataires (René et Panet-Raymond, 1984). Au début des années 1970, l'Association des locataires du Montréal métropolitain obtient une centaine des postes de salariées et salariés pour différentes associations de quartier dont le travail s'oriente vers le service et le dépannage, c'est-à-dire l'aide individuelle et directe de locataires en difficulté (René et Panet-Raymond, 1984). Puis, à la suite de la coupure du « robinet à piastres » du gouvernement fédéral, la presque totalité de ces associations disparaît en 1974 (René et Panet-Raymond, 1984). 
Au cours des années 1970, de nouveaux comités logement et associations de locataires émergent grâce à l'implication bénévole de citoyennes et citoyens. C'est ainsi que naissent, entre autres, le Comité logement Centre Sud ${ }^{2}$ en 1976, l'Association des locataires de Villeray (ALV) ainsi que le Comité logement de Rosemont en 1977. Ces comités mettent sur pied des services autogérés d'information ayant pour objectif de permettre aux locataires de se défendre contre les abus de certains propriétaires. En plus de donner de l'information juridique, ils offrent une aide technique dans la rédaction de communications ainsi qu'un accompagnement physique et moral visant à sécuriser les locataires tout au long de leurs démarches légales. Ces groupes exercent également des pressions politiques, déploient des actions à dimension collective et dérangent l'ordre public afin d'attirer l'attention sur certains enjeux locaux. De telles actions visent, par leur caractère sociopolitique, à promouvoir et à défendre des droits sociaux ainsi qu'à réclamer des lois et des programmes plus justes et plus équitables (Duval, et collab., 2005).

"On compte aujourd'hui, à l'échelle du Québec, plus de 45 groupes de défense de droits des locataires, comités de citoyennes et citoyens avec un volet logement ou encore services de référence en matière de logement au sein d'organismes ou de tables de concertation ${ }^{3}$."
On compte aujourd'hui, à l'échelle du Québec, plus de 45 groupes de défense de droits des locataires, comités de citoyennes et citoyens avec un volet logement ou encore services de référence en matière de logement au sein d'organismes ou de tables de concertation ${ }^{3}$. À Montréal seulement, où plus de 487000 ménages sont locataires (Ville de Montréal, 2009, p. 6), une vingtaine de groupes ${ }^{4}$ poursuivent à la fois cette mission de renseigner les personnes locataires sur leurs droits, leurs recours et leurs obligations ainsi que de promouvoir le logement social.

La plupart des groupes adoptent un discours opposant les questions de logement social et celles de logement privé , et ce, généralement en raison des dynamiques créées par les regroupements dont ils sont membres, soit le Regroupement des comités logement et associations de locataires du Québec (RCLALQ) et le Front d'action populaire en réaménagement urbain (FRAPRU). Le premier se donne notamment comme mission la défense des droits des locataires par la formation, l'information et la diffusion du contenu juridique, politique et social lié au marché locatif, alors que le second lutte pour la promotion des droits sociaux et considère le logement comme un bien auquel tous devraient avoir accès par l'intermédiaire d'une intervention étatique. Comme nous le verrons, les travailleuses et travailleurs des groupes rencontrés ne voient pas d'incompatibilités entre ces deux sphères du logement, puisque ce sont généralement les 


"... il nous apparaît qu’il
convient de poser un regard
actualisé sur les enjeux entou-
rant les idéaux d'autonomie
au sein des comités loge-
ment qui simposent à la fois
comme ressources expertes
sur les questions locatives,
espaces de représentation des
personnes locataires et lieux
de développement du pouvoir
d'agir. "
dont six sont de récents diplômés ou diplômées universitaires ayant de 25 à 35 ans. Les sept personnes répondantes travaillent dans sept différents organismes de défense des droits des locataires depuis au moins deux ans, et trois d'entre elles ont une formation en travail social, les quatre autres ayant fait des études dans des disciplines connexes en sciences humaines. de défense de droits des personnes locataires, il nous apparaît qu'il convient de poser un regard actualisé sur les enjeux entourant les idéaux d'autonomie au sein des comités logement qui s'imposent à la fois comme ressources expertes sur les questions locatives, espaces de représentation des personnes locataires et lieux de développement du pouvoir d'agir.

Pour ce faire, nous avons mené des entrevues semidirigées ( $\mathrm{n}=7$ ) d'environ 60 minutes au printemps 2013 et à l'automne 2015 avec des travailleuses et travailleurs d'organismes communautaires en logement de la région de Montréal. Il s'agit de deux hommes et cinq femmes, difficultés vécues par les locataires habitant un logement privé qui les amènent, dans un premier temps, à prendre connaissance de l'existence des groupes de défense de droits pour, dans un deuxième temps, devenir demandeurs d'un logement social. La majorité des activités des comités logement sont donc planifiées en fonction des plans d'action de leurs regroupements.

Ces organisations, dont on reconnaît l'apport à la santé et au bien-être de la population en tant que dispensateurs qualifiés de services depuis plus de 25 ans $^{6}$, sont aujourd'hui financées par le Secrétariat à l'action communautaire autonome et aux initiatives sociales (SACAIS). Certaines perçoivent également des fonds de Centraide ${ }^{7}$, touchent la contribution secteur de la Société d'habitation du Québec (SHQ) ainsi que des sommes des communautés religieuses. Plus de quarante ans après la création des premiers groupes de défense de droits des locataires, nous souhaitons proposer un regard critique sur leurs modes d'appréhension de l'idéal démocratique en regard des limites organisationnelles existantes ${ }^{8}$.

\section{L'organisation communautaire et l'approche sociopolitique}

Quarante ans après l'émergence des premiers groupes . 
Le recrutement des répondantes et répondants a été basé sur une méthode non probabiliste. Nous avons dressé une liste de tous les comités logement, puis nous avons communiqué avec chacun d'entre eux pour sonder l'intérêt d'un travailleur ou d'une travailleuse à participer à l'étude, généralement la personne qui a pris le message et qui nous a rappelée. Le seul critère de recrutement était d'avoir un minimum de deux ans d'expérience dans un organisme de défense de droits de locataires. Bien que le nombre d'entretiens réalisés puisse sembler limité, les répondantes et répondants proviennent de sept groupes communautaires différents, ce qui laisse supposer qu'il s'agit d'une perspective assez représentative de l'ensemble, car on dénombre dixsept comités logement au total'

Afin de saisir la position de l'intervention sociale dans ses pratiques démocratiques, les personnes répondantes ont été invitées, dans un premier temps, à décrire puis à commenter la mission et les stratégies d'intervention présentes dans leur organisme, et à évaluer les modèles d'intervention privilégiés à partir d'une perspective critique. Puis, dans un deuxième temps, elles ont été questionnées sur la place accordée aux militantes et militants au sein de leurs organismes, aux espaces de délibération et aux relations qui caractérisent les interactions entre les militantes et militants et les travailleuses et travailleurs. Dans un troisième temps, elles ont été invitées à identifier les principaux enjeux qu'elles rencontrent et à dresser un bilan des limites organisationnelles qu'elles perçoivent. L'analyse des résultats s'est effectuée à partir des étapes d'analyse de contenu que propose L'Écuyer (1987) : lecture préliminaire et établissement d'une liste, découpage du matériel, réorganisation du matériel, description des résultats basée sur l'analyse qualitative, la quantification et le traitement statistique, et interprétation des résultats.

Pour analyser les modes d'appréhension de l'idéal démocratique visant le développement de l'autonomie des personnes participantes au sein des groupes de défense de droits des locataires, les militantes et militants, rappelons d'abord les fondements de l'organisation communautaire. Cette pratique repose sur l'idée fondatrice selon laquelle les problèmes sociaux sont de nature collective et requièrent, par conséquent, des solutions collectives. Elle se propose d'être un moyen de promotion de la justice sociale et de remise en question des relations de pouvoir et des privilèges dont jouissent les membres d'une minorité et une tribune afin que les " dépossédés " puissent faire entendre leur voix (Shragge, 2006, p. 51).

L'organisation communautaire se rattache à quatre principales stratégies d'action que sont l'approche socioéconomique (développement local), l'approche sociopolitique (action sociale), l'approche socio-institutionnelle (planning social) et l'approche sociocommunautaire (entraide et services de proximité) (Bourque, et collab., 2007). Celle sur 
laquelle se sont appuyés les groupes de défense des locataires au moment de leur création relève de l'approche " sociopolitique " (René et Panet-Raymond, 1984). Cette approche stratégique propose de résoudre des problèmes sociaux perçus et ressentis comme des injustices par la défense et la promotion de droits sociaux. Les organisations qui les combattent prennent la forme de groupes de lutte et sont porteuses de revendications et de pressions du registre de l'action directe allant du conflit au compromis avec les

"Ce mode d'intervention reconnaît aux citoyennes et citoyens le droit de sorganiser, de revendiquer et de sinscrire dans le rapport social de forces de leur société..." autorités, et ce, dans l'objectif de développer des structures autonomes de type syndical (Bourque, et collab., 2007). Ce mode d'intervention reconnait aux citoyennes et citoyens le droit de s'organiser, de revendiquer et de s'inscrire dans le rapport social de forces de leur société (Bourque, et collab., 2007, p. 18) afin de traduire leurs frustrations et leurs désirs en droits, de les codifier, puis de les faire respecter (Wyers, 1991).

L'approche sociopolitique, par conséquent, accorde une place prépondérante à la dimension politique et au conflit. Ce dernier fait partie de la dynamique sociale, étant donné les rapports et les intérêts divergents entre les classes sociales, et apparaît également comme un moyen de faire pression pour négocier le changement souhaité (Comeau, 2007, p. 82). Ce cadre de référence s'appuie sur une lecture marxiste qui tire sa source dans le courant politique de l'époque, lequel situe les interactions dans une lutte des classes opposant les locataires — qui sont les petits travailleurs, les chômeurs et les citoyens et qui constituent les classes populaires - et les propriétaires, les bourgeois et l'État — qui représentent le capitalisme. L'analyse des entrevues menées permettra d'identifier de quelles façons cette approche prend forme au quotidien et quelles en sont les limites, voire les paradoxes.

\section{Le bilan des pratiques : la rencontre entre le théorique et la pratique}

Selon Mayer, Lamoureux et Panet-Raymond (2008), l'action communautaire au Québec se veut le lieu de l'expression de la capacité de la population de s'organiser collectivement en vue de proposer des changements sociaux. Or, les entrevues réalisées avec des travailleuses et travailleurs d'organismes communautaires nous permettent de constater qu'il existe un fort décalage entre les approches théoriques véhiculées et la capacité d'aménager des lieux permettant leur réalisation. Ainsi, comme le soulignent Guberman, et collab. (1994), le 
tout n'est pas simplement une question de volonté ou de capacité des intervenantes et intervenants à vulgariser leurs propos et à s'adapter au rythme des militantes et militants. La complexité des enjeux et la nature des débats peuvent constituer des limites importantes aux possibilités d'implication et à la participation aux processus décisionnels pour les destinataires de l'organisme. Il existe une certaine tension entre, d'une part, les objectifs d'éducation populaire et, d'autre part, les exigences d'un fonctionnement efficace et de qualité d'un organisme. À cette première tension s'ajoute une seconde qui concerne l'imbrication entre les valeurs d'égalitarisme et de non-hiérarchisation entre militants et travailleurs, et une tendance à la professionnalisation, qui implique que les intervenantes et intervenants possèdent une formation universitaire, une expertise et des compétences, ce que n'ont pas nécessairement les destinataires de l'organisme.

\section{Mandat, mission et rôle des travailleuses et travailleurs}

Selon le Rapport de l'enquête du comité de direction auprès des groupes membres du Regroupement pour le gel des loyers de 1979, l'objectif premier d'un comité logement était, à la fin des années 1970, d'organiser les locataires d'un quartier pour la défense de leurs droits et de leurs intérêts (Milot, 1979, p. 1). À l'époque, le statut de locataire désignait également celui de travailleur, de sorte que la lutte sur le front du logement était avant tout celle de la classe ouvrière. Cette vision s'appuyait sur une lecture marxiste des conditions de vie des couches populaires. Cette mission se réalise dans l'éducation sur la situation locative, sur ses responsables et ses causes, sur les enjeux et les luttes à mener, dans l'information quant aux droits et aux mécanismes de défense, ainsi que dans

"Les travailleuses et travailleurs rencontrés, qui portent ou ont porté le titre d'organisateur ou organisatrice communautaire ${ }^{11}$, décrivent leur travail comme étant celui d'informer les locataires, de les regrouper, de les mobiliser, d'animer la vie associative et de favoriser le développement de liens de solidarité..." la construction d'une unité dans les luttes populaires et ouvrières.

Cette conception de la mission des comités logement semble encore être celle partagée par les travailleuses et travailleurs que nous avons rencontrés. Tous s'entendent pour définir leur organisme comme étant un groupe de défense collective de droits qui, ne s'adressant qu'aux personnes locataires ${ }^{10}$, travaille à l'amélioration des conditions de vie ainsi qu'à l'amélioration de la qualité de vie des citoyennes et citoyens de leur quartier, et ce, par l'intermédiaire d'activités d'éducation populaire et d'activités de mobilisation. 
Les travailleuses et travailleurs rencontrés, qui portent ou ont porté le titre d'organisateur ou organisatrice communautaire ${ }^{11}$, décrivent leur travail comme étant celui d'informer les locataires, de les regrouper, de les mobiliser, d'animer la vie associative et de favoriser le développement de liens de solidarité, c'est-à-dire, "dans les mots de [leurs] bailleurs de fonds, de favoriser l'implication citoyenne des gens du quartier». Travaillant toutes et tous à partir d'un modèle d'intervention basé sur la défense de droits, les répondantes et répondants endossent une diversité de rôles qui les positionnent à la fois en tant que vulgarisateur, facilitateur, analyste et porte-parole :

"Mon travail m’amène à agir à la fois comme une experte, mais aussi comme un chien de garde. » (Entrevue 7)

Tous les organismes pour lesquels travaillent les répondantes et répondants ont deux grands volets d'intervention : la défense individuelle des droits des locataires, qu'on appelle le "service aux locataires ", et la défense collective des droits, qui passe essentiellement par la promotion du logement social. La défense individuelle des droits, appelée le " service aux locataires » ou " l'accueil des locataires », prend la forme de services-conseils juridiques dispensés au sein de l'organisme par un ou plusieurs membres de l'équipe de travail n'ayant pas, sauf exception, de formation juridique. L'aide peut être offerte par téléphone ou lors de rencontres selon une formule avec ou sans rendez-vous. Les services offerts sont la vulgarisation de lois et de règlements municipaux, l'information sur les droits, obligations et recours des locataires, la rédaction de mises en demeure, l'aide dans le dépôt d'une demande de logement social, la référence vers d'autres ressources psychosociales et l'aide dans l'ouverture d'un dossier ou le dépôt d'une plainte à la Régie du logement ${ }^{12}$ et à la Ville de Montréal. Certains groupes offrent également un service d'accompagnement, de visite des logements et d'intervention auprès des propriétaires.

"En individuel, on travaille dans une optique de répondre aux besoins des locataires. On fait le plus possible les démarches avec eux. On est beaucoup dans l'informel, on ne travaille pas avec un plan d'intervention. On essaie de s'adapter le plus possible pour que la personne ait le plus de chances de défendre ses droits. » (Entrevue 5)

Les travailleuses et travailleurs reconnaissent un certain aspect " clientéliste » dans l'approche de défense individuelle de droits, mais insistent sur l'idée selon laquelle l'information est une première étape d'une démarche d'appropriation de ses conditions de vie pouvant éventuellement mener à l'engagement dans des luttes. Un répondant mentionne également que les gens viennent chercher de l'aide, sans toutefois trop 
comprendre à quel type d'organisation ils s'adressent, ni quels sont ses buts sous-jacents, mais surtout qu'on ne prend pas le temps de leur expliquer :

« Ici, à l'accueil, il y a un rapport clientélisme. Les gens viennent avant tout pour se faire aider ou en pensant qu'ils vont obtenir un logement en venant chez nous. Souvent, ils peuvent confondre. Ils peuvent ne pas savoir c'est quoi un organisme communautaire, ne pas savoir qu' ils peuvent être membres, que ce sont les citoyens qui théoriquement contrôlent. C'est une information que les gens n'ont pas d'emblée. " (Entrevue 3)

Par ailleurs, on précise que ce ne serait toutefois qu'une mince minorité des interventions au service d'information juridique qui déboucheraient sur une prise de conscience du caractère collectif et social des problèmes locatifs, ou encore sur l'implication de la personne locataire dans les activités de l'organisme.

"Si tout ce qu'on fait, c'est d'informer les gens sur leurs droits et les préparer pour leur audience à la Régie, alors que la Régie, c'est de la grosse marde, et que, collectivement, on ne se lève pas pour dire que la Régie, ça ne marche pas, ça ne sert à rien d'être dans la défense individuelle. » (Entrevue 4)

"Le jumelage de l'intervention individuelle à l'intervention collective alimente une polémique depuis le tout début des comités logement..."

Le jumelage de l'intervention individuelle à l'intervention collective alimente une polémique depuis le tout début des comités logement (René et Panet-Raymond, 1984, p. 76-77). Toujours sensibles à cette controverse, les répondantes et répondants repoussent l'idée d'opposition ou encore de dichotomie entre l'intervention individuelle et l'intervention collective. À leur avis, le service est une pratique d'intervention complémentaire aux objectifs de lutte des groupes. Elle est décrite comme la "porte d'entrée " des locataires dans leur comité, c'est-à-dire comme une "façon d'aller rejoindre les gens qui ont des problèmes ». Les travailleuses et travailleurs insistent toutefois sur l'importance que le service d'information juridique aux locataires ne transforme pas le groupe de défense de droits en une " annexe » ou un " guichet de service » de la Régie du logement qui doit suppléer à l'incapacité de cette dernière dans sa mission de renseigner les locateurs et les locataires sur leurs droits et obligations résultant du bail d'un logement ${ }^{13}$. Mise sur pied en 1979 à la suite de la publication du Livre blanc sur les relations entre locateurs et locataires, la Régie du logement a été aménagée pour contrer l'inefficacité du partage de la juridiction entre les tribunaux civils de l'époque, la complexité de leurs procédures, les frais judiciaires et extrajudiciaires occasionnés, et 
les délais requis avant l'obtention d'un jugement. Les auteurs du rapport avaient tablé sur la nécessité que des moyens incitatifs et coercitifs soient déployés afin de " rétablir l'équilibre entre les parties en présence, en assurant à chacun non seulement des droits mais aussi des recours équivalents ", soit des " recours simples, efficaces et rapides ", ainsi qu' « une information adéquate et continue» (gouvernement du Québec, 1978, p. 15). Notons que moins d'un an après la création de la Régie, elle faisait déjà l'objet de critiques virulentes ${ }^{14}$ qui lui sont encore adressées aujourd'hui.

"Les travailleuses et travailleurs exposent le caractère collectif de la démarche de défense de droits dans laquelle leur organisme sinscrit et qui fait référence à la lutte. "
Les travailleuses et travailleurs exposent le caractère collectif de la démarche de défense de droits dans laquelle leur organisme s'inscrit et qui fait référence à la lutte. La défense collective est présentée non pas comme un ensemble de pratiques d'intervention, mais bien comme un mode d'appréhension de la réalité dans lequel les abus et les dénis de droits subis par les locataires individuellement se doivent d'être analysés non pas en tant que problèmes isolés, mais
nes systémiques nécessitant des solutions systémiques. Les bien en tant que phénomènes systémiques nécessitant des solutions systémiques. Les
stratégies revendicatrices actuelles s'inspirent donc des mêmes idéologies que celles qui caractérisaient la fin des années 1970, alors qu'elles empruntaient des couleurs très politiques en s'attaquant directement à l'État et en exigeant des réformes radicales et immédiates (René et Panet-Raymond, 1984, p. 8).

La défense collective des droits prend essentiellement la forme de mobilisations autour des questions reliées à la justice sociale, à la pauvreté et à la lutte pour le maintien ainsi que l'amélioration du financement du logement social. Le logement social désigne les coopératives d'habitation, les OBNL d'habitation et les habitations à loyer modique (HLM), trois modèles d'habitation représentant une alternative au marché privé et à ses impératifs de rentabilité et de profits. Il offre, grâce au financement de l'État, des logements à des coûts plus adaptés à la capacité de paiement des locataires tout en leur permettant de s'impliquer à diverses échelles dans la gestion de leur milieu de vie. Il vise donc essentiellement l'amélioration de la qualité de vie des personnes locataires.

Les approches de mobilisation privilégiées décrites sont sensiblement les mêmes d'une année à l'autre : manifestations, actions tintamarres, pétitions, occupations, etc. Elles visent la création d'un rapport de force avec l'État, et avec ses élues et élus, pour que soient modifiées les politiques et orientations gouvernementales afin qu'elles soient plus sensibles aux besoins des personnes les plus démunies. Toutefois, les répondantes et répondants, à l'exception d'une, mentionnent que ce rapport de force, malgré tous les 
efforts qui sont investis dans sa construction, n'est que trop peu souvent atteint. Pour certains des répondants et répondantes, la poursuite quasi aveugle de cet idéal alimente trop souvent des pratiques qui auraient pour résultat d'escamoter les étapes de base de l'organisation communautaire :

"Souvent, on veut amener les gens vers des actions plus grandes, on veut lancer un journal et on va faire ça devant des condos avec une conférence de presse, alors que, des fois, on oublie les affaires de base comme faire du café, commander de la pizza. Souvent, on oublie des affaires de base, on est plutôt portés à planifier des affaires et après à appeler notre monde pour remplir les autobus.» (Entrevue 3)

"On bourre le crâne des gens. On essaie plus de convaincre les gens que d'aller chercher ce qu'eux voient comme solution. " (Entrevue 7)

L'une des répondantes insiste sur la nécessité que les organismes remettent en question leurs façons de faire, mais ils ne le font pas parce qu'ils se sentent dépassés par l'ampleur de leur travail et se retrouvent accablés d'une certaine inertie :

"C'est comme si notre boîte à outils, on a quatre outils dedans, et c'est toujours les mêmes qu'on utilise, de la même façon [...] J'ai l'impression qu'il y a un blocage. On fait ça depuis longtemps, on ne se pose pas vraiment de questions, on fait juste continuer à faire ça et, des fois, on est fatigués et on est tannés. On se rend compte que ce n'est pas super efficace, mais changer les habitudes, c'est souvent déstabilisant, épeurant, menaçant, difficile à imaginer. " (Entrevue 2)

Les répondantes et répondants reconnaissent que les victoires dans le milieu du logement sont "petites » et sont gagnées "à l'arraché ». Soulignons à titre d'exemples : que le gouvernement fédéral n'investit plus dans la construction du logement social depuis 1994; qu'il n'existe toujours pas de code de logement provincial; que la Régie du logement, qualifiée de "système de justice dérisoire ${ }^{15}$ dès sa création, est toujours la cible de campagnes virulentes en raison de ses délais d'attente pour l'obtention d'une première audience ${ }^{16}$, délais qui lui ont valu le surnom de Régie du lentement ${ }^{17}$ et de Mépris du logement ${ }^{18}$; qu'il existe toujours des pratiques illégales discriminatoires ou liées à la collecte de renseignements personnels non nécessaires lors de la recherche de logement; que le parc de logements locatifs n'est toujours pas sous le coup d'une protection ou d'un moratoire permettant d'éviter sa commercialisation. 
"... les répondantes et répondants estiment que leurs actions conduisent à l'amélioration de la connaissance des droits des personnes locataires et leur permettent de développer des outils pour s'affirmer et éviter les abus et dénis de droits. "

Bien qu'il ne soit pas possible de mesurer avec précision les retombées des pratiques d'intervention des comités logement, les répondantes et répondants estiment que leurs actions conduisent à l'amélioration de la connaissance des droits des personnes locataires et leur permettent de développer des outils pour s'affirmer et éviter les abus et dénis de droits. Ces personnes peuvent ensuite améliorer leurs conditions de vie ou, du moins, éviter qu'elles ne se dégradent. Parmi les autres "victoires", on souligne que des changements législatifs ont eu lieu ainsi que des terrains pour le développement du logement social ont été obtenus. Un des répondants ajoute que de dresser un bilan est une réflexion plutôt difficile puisque la plupart des luttes actuelles portent sur le maintien des " acquis sociaux ", c'est-à-dire des programmes gouvernementaux d'aide aux plus démunis : supplément au loyer, aide sociale, assurance-emploi, financement des coopératives d'habitation, etc. On souligne donc que cette situation où, d'une part, les gains ne sont ni visibles ni connus parce qu'ils ne sont pas suffisamment documentés et diffusés et où, d'autre part, la lutte est orientée non pas vers l'obtention de gains mais vers le maintien des acquis, occasionne à la fois du découragement et une démobilisation.

En somme, les travailleuses et travailleurs insistent sur le caractère longitudinal de la défense collective de droits et le changement de système qu'elle impose, un changement qui nécessite une révolution dans les façons d'appréhender le monde et ses problèmes. Un des répondants nous met toutefois en garde de tomber dans ce qu'il appelle l'" approche collective sans citoyen ", c'est-à-dire dans une rhétorique où l'énergie se concentre dans les discussions théoriques sur la meilleure approche à adopter, pour finalement ne faire ni service, ni mobilisation.

\section{Structure organisationnelle et place aux militantes et militants}

Selon les répondantes et répondants, les locataires qui s'impliquent dans les comités logement sont d'anciens travailleurs, travailleuses ou stagiaires de l'organisme, d'anciens militants et militantes de groupes d'idéologie dite de gauche, des locataires en attente d'un logement social ou encore des locataires qui ont fait appel à l'organisme pour avoir de l'aide dans leur situation locative. On souligne que bien peu de militantes et militants qui s'impliquent dans les comités ont une compréhension approfondie et une vision articulée 
des enjeux locatifs, mais qu' elles et ils sont davantage dans une dynamique de "support " du travail des personnes salariées avec qui elles et ils entretiennent un lien de confiance :

«Il y a beaucoup de locataires qui sont devenus membres pour nous appuyer parce qu'ils ont reçu un service [...] Le conseil d'administration est composé de gens qui ne se posent pas de questions sur ce qui serait le mieux pour l'organisme. Ils prennent beaucoup de choses de la part de la direction comme du cash. » (Entrevue 5)

"...pourquoi la plupart des répondantes et répondants expliquent que la place donnée aux militantes et militants dans leur organisme est fortement discutable, voire contestable, car elle ne correspond pas aux idéaux de développement du pouvoir d'agir véhiculés."

Cette situation ne correspond néanmoins pas à la vision idéale de la participation qu'entretiennent les travailleuses et travailleurs rencontrés. C'est pourquoi la plupart des répondantes et répondants expliquent que la place donnée aux militantes et militants dans leur organisme est fortement discutable, voire contestable, car elle ne correspond pas aux idéaux de développement du pouvoir d'agir véhiculés. On critique le traitement réservé aux militantes et militants :

"La participation, c'est pas : vient faire le mailing quatre fois par année, mets ton nom pour cuisiner la soupe une fois par mois, mets ton nom quand on sort pour une manif callée par un des regroupements [...] Il est où le réel espace où les gens peuvent dire : moi, j’ai observé ça dans le quartier, ça me préoccupe, et j'aimerais ça qu'on travaille là-dessus? » (Entrevue 4)

"On donne très peu de place aux membres. On compte sur eux pour la participation dans les manifestations, mais on ne les consulte pas dans ce qui se fait en amont. C'est rare qu'on les implique dans la prise de décisions. C'est plus : on fait telle chose, venez-vous-en! » (Entrevue 7)

"Malgré nos idées d'éducation populaire, de travailler avec les gens, de prendre le temps, d'avoir des espaces pour discuter de ces questions... On accorde moins d'importance à des propos qu'on va juger périphériques, s'ils ne correspondent pas à notre cadre idéologique et théorique. » (Entrevue 6)

À l'exception d'une répondante, les travailleuses et travailleurs jugent que leur organisme connait d'importantes faiblesses en termes de démocratie interne et expliquent 
cette situation par la concentration du pouvoir entre les mains des équipes de travail. La mobilisation se trouve donc limitée par les structures mêmes de l'organisation qui ne facilitent pas la mobilisation, soit parce qu'elles sont inexistantes, soit parce qu'elles ne permettent pas une réelle prise en compte des prises de parole des militantes et militants. À ce sujet, on déplore notamment que les plans d'action soient rédigés par les équipes de travail à huis clos, que les assemblées générales soient des simulacres de démocratie où les orientations sont déjà décidées et que les équipes de travail aient le pouvoir de proposer ou non des actions à leurs membres :

"Oui, on leur présente un plan d'action lors de l'AGA, mais c'est quelque chose qui a déjà été réfléchi. On arrive avec nos choses, ce ne sont pas les membres qui pensent. C'est plus du rubberstamping. "
"Oui, on leur présente un plan d'action lors de l'AGA, mais c'est quelque chose qui a déjà été réfléchi. On arrive avec nos choses, ce ne sont pas les membres qui pensent. C'est plus du rubberstamping. » (Entrevue 7)

«En fait, il n'y a quasi aucune possibilité sur place pendant l'AGA d'apporter des propositions. On ne fait pas voter des plans d'action, on ne fait pas voter des mandats de grève. On présente seulement nos grandes orientations qui ne disent rien. » (Entrevue 5)

C'est pourquoi les travailleuses et travailleurs insistent sur le besoin de multiplier les espaces où les locataires peuvent contribuer aux réflexions, à l'élaboration des décisions et à l'organisation des actions.

«Si on n'ouvre jamais cette possibilité-là, elle n'existera jamais [...] On veut que les citoyens développent leur pouvoir d'agir, mais on ne leur donne pas de place pour qu'ils développent ce pouvoir d'agir là. C'est un tournant qui est nécessaire. Sinon, c'est quoi notre raison d'être? » (Entrevue 5)

Cinq des répondantes et répondants rapportent l'existence de distinctions créées par les équipes de travail entre les termes utilisés pour décrire les personnes qui s’impliquent dans les organismes : certains comités réservent le terme militant aux gens qui participent aux actions directes, puisque " ceux-là ont une étiquette qui vient avec une couronne d'or ", et accolent le titre de bénévole aux personnes qui font des tâches internes comme les appels téléphoniques, les envois postaux et la préparation de la nourriture. Les répondantes et répondants dénoncent ces façons de faire puisqu' elles engendrent une hiérarchisation méritoire des formes d'implication et accentuent une déjà existante division sexuée des 
tâches où les femmes, bénévoles, s'occupent de choses futiles pendant que les hommes, militants, sortent dans les rues afin de poser de vraies actions.

Dans leur idéal d'intervention, les travailleuses et travailleurs n'agiraient qu'en tant que facilitateur auprès des membres. Un des répondants recommande d'impliquer les militantes et militants dans le quotidien des groupes en leur donnant la possibilité d'effectuer des tâches qui sont normalement sous la responsabilité de l'équipe de travail : animation d'ateliers, service d'information aux locataires, etc., afin de leur donner « la place qui leur revient $"$ :

«Une personne qui part d'un problème de logement, qui a de la misère à en parler et à se débrouiller avec ça peut, en bout de ligne, faire valoir ses droits et enseigner à d'autres personnes comment défendre leurs droits. Là, je pense qu'on a atteint de quoi de gros. » (Entrevue 1)

Cette façon de faire n'est toutefois pas partagée par tous les répondants et répondantes qui jugent que leur organisme nécessite une profonde remise en question des pratiques ainsi qu'une analyse des rapports de pouvoir présents.

Les répondantes et répondants soulignent que la façon dont on accueillera ou répondra à une idée, à une préoccupation qui est soulevée ou à une suggestion d'activité, aura une influence directe sur la perception que les militantes et militants auront de leur rôle ainsi que de celui des personnes salariées. Le pouvoir décisionnel peut ainsi rester dans les mains des salariées et salariés :

«Le problème le plus important, c'est que l'équipe de travail a de facto un pouvoir énorme, a le pouvoir de contacter les gens ou de ne pas les contacter, a le pouvoir de dire on propose cette action-là ou encore, ah non, cette actionlà, ça ne nous tente pas, on ne la fait pas. Tout est une question de comment on utilise le pouvoir qu'on a. Et ça, ça va dépendre de comment on voit les gens avec qui on travaille. " (Entrevue 2)

"Est-ce qu'on représente vraiment la volonté des citoyens? [...] Toute la critique, par exemple PSL ${ }^{19}$ privé. Dans notre vision, ce n'est pas dans cette direction que l'État devrait aller, mais quand tu parles aux locataires, les gens voudraient leur PSL privé. Les gens sont pas mal critiques des coops et aimeraient mieux rester dans leur duplex, triplex. Mais, tu ramènes la ligne contre le PSL privé. » (Entrevue 6) 
L'exemple de la prise de position sur le PSL qui est mentionné lors d'une entrevue est particulièrement intéressant, car il confronte des intérêts idéologiques à des intérêts individuels. Le discours porté par les organismes et leurs regroupements, en particulier le FRAPRU ${ }^{20}$, est que le supplément au loyer privé, contrairement au logement social, ne permet ni la construction de nouveaux logements, ni leur revitalisation. Encore pire, il ne permet d'aucune façon de sortir le logement locatif de la logique du profit

"Toujours selon ce discours, il serait préférable qu'une personne locataire désirant accéder au proloyer fasse une demande de logement social, ce qui signifie qu'elle doive quitter le logement qu'elle occupe pour avoir une aide au paiement de son loyer. " gramme de Supplément au

en subventionnant directement des propriétaires de logements privés. Toujours selon ce discours, il serait préférable qu'une personne locataire désirant accéder au programme de Supplément au loyer fasse une demande de logement social, ce qui signifie qu'elle doive quitter le logement qu'elle occupe pour avoir une aide au paiement de son loyer. La formule du PSL privé, quant à elle, permet que la subvention soit versée à un propriétaire d'un logement privé et n'implique donc pas nécessairement et systématiquement un déménagement et un déracinement. Ainsi, bien que des locataires et des membres puissent juger que le PSL privé soit une solution davantage adaptée à leurs besoins, les intervenantes et intervenants dénoncent cette forme de privatisation d'aide, car elle ne correspond pas aux revendications de lutte ni aux idéaux véhiculés.

De plus, bien que les comités logement portent l'objectif de valoriser l'autonomie des individus, ils semblent incapables de mettre en œuvre cette volonté en raison notamment du poids de leurs contraintes extérieures (" faire plus avec moins»). Comme le mentionne Fontaine (2013), les attentes de rendement imposées aux organismes communautaires par les bailleurs de fonds les poussent à se préoccuper davantage des résultats que de l'autonomie de leurs participantes et participants, qui ne sont que relativement peu impliqués dans les espaces de délibération de l'organisme. Dans un contexte de sous-financement des organismes communautaires autonomes, et particulièrement des organismes de défense collective de droits, une des répondantes explique que les contraintes de financement ont pour effet de créer beaucoup de mobilité dans l'équipe de travail.

« Le financement par projet joue beaucoup sur notre capacité à tisser des liens avec les membres. Ça prend du temps aux gens pour faire confiance à une personne, puis elle s'en va.» (Entrevue 7)

En somme, il apparaît que la place accordée aux militantes et militants dans les espaces de délibération, ainsi que dans l'orientation des actions, alimente d'importants 
questionnements idéologiques et professionnels chez les travailleuses et travailleurs que nous avons rencontrés. Ces travailleuses et travailleurs dénoncent même, dans une certaine mesure, l'instrumentalisation des militantes et militants de leur organisme, qui se retrouvent à remplir des autobus sans qu'on leur ait donné la parole afin de savoir ce qu'elles et ils souhaitent faire et comment elles et ils veulent le faire.

\section{Conclusion}

S'inspirant d'une approche " par le bas $"^{21}$, l'organisation communautaire vise le rassemblement de personnes afin qu'elles luttent ensemble pour résoudre des problèmes "La stratégie d'action privilégiée par les comités logement, soit l'approche sociopolitique, repose sur un travail de promotion et de défense des droits qui passe par la lutte, le port de revendications et l'exercice de pressions afin de développer un rapport de forces plus équilibré. » communs, dont les inégalités, la concentration du pouvoir et les discriminations, et qu'elles s'impliquent dans la prise des décisions qui les affectent. La stratégie d'action privilégiée par les comités logement, soit l'approche sociopolitique, repose sur un travail de promotion et de défense des droits qui passe par la lutte, le port de revendications et l'exercice de pressions afin de développer un rapport de forces plus équilibré. Elle s'appuie sur une vision politique selon laquelle les problèmes sont de nature collective et nécessitent, par conséquent, des solutions collectives; l'émancipation individuelle s'atteint par la transformation collective.

Les intervenantes et intervenants rencontrés sont ouverts au jumelage entre l'intervention individuelle et l'intervention collective, mais semblent particulièrement perplexes devant les obstacles à surmonter dans la conciliation de la diversité de leurs rôles. Elles et ils jouent, tour à tour, les rôles d'analyste, vulgarisateur, plaideur, organisateur, lobbyiste, politicien, mais aussi d'ami, confident et militant. Elles et ils semblent se retrouver ensevelis sous une multitude de rôles, de responsabilités et de contraintes, mais également d'idéaux, et apparaissent particulièrement conscients de la difficile articulation entre le tout.

C'est pourquoi, comme nous l'avons vu, les travailleuses et travailleurs se montrent insatisfaits face aux pratiques d'intervention déployées au quotidien dans leur milieu de travail. Notre analyse démontre que six des sept répondantes et répondants entretiennent une vision extrêmement critique quant aux pratiques de participation interne qui caractérisent leur organisme. Elles et ils dénoncent essentiellement les inéquations entre 
leurs idéaux et le nonaccès aux espaces décisionnels des personnes locataires impliquées au sein de leurs groupes, ainsi que la concentration du pouvoir entre les mains des équipes de travail, soit des personnes rémunérées. Les répondantes et répondants estiment que cette situation résulte partiellement d'une certaine inertie et de l'absence de remises en question collectives des pratiques d'intervention dans les groupes.

Les réflexions présentées dans cet article s'avèrent particulièrement fécondes et démontrent à quel point, quarante ans après la création des premiers comités logement, le moment semble être des plus opportun pour faire collectivement le point sur leurs pratiques d'intervention, et ce, notamment en ce qui concerne la place que l'on donne aux militantes et militants, ou celle que l'on veut bien leur donner. En effet, ces personnes doivent être réintégrées au sein même des instances décisionnelles des organismes afin que ces derniers les représentent réellement et ne soient pas seulement des porteurs d'idéaux théoriques. À l'instar du programme de Supplément au loyer (PSL) privé, la question de l'interdiction des plates-formes d'hébergement touristique (dont AirBnB est la plus connue) est aujourd'hui au centre des luttes des comités logement des quartiers centraux montréalais. Les intervenantes et intervenants sont porteuses et porteurs de discours dénonçant le fait que ces plates-formes réduisent l'offre de logements locatifs abordables, qu'elles participent à l'augmentation du prix des loyers, qu'elles compromettent la vie du quartier, qu'elles causent des troubles de voisinage en raison des bruits excessifs et qu'elles favorisent le phénomène de la gentrification. Plusieurs membres toutefois ne voient pas nécessairement de mal à louer une chambre occasionnellement pour obtenir de petits revenus supplémentaires et pensent qu'il vaudrait mieux encadrer ce qui est illégal. Les travailleuses et travailleurs consacrent ainsi beaucoup de leur temps à mobiliser "Les travailleuses et travailleurs consacrent ainsi beaucoup de leur temps à mobiliser les militantes et militants avec la "ligne dure " de la nécessité d'interdiction de l'hébergement touristique, sans nécessairement que ce soient des positions votées par les membres et avec lesquelles elles et ils sont d'accord." les militantes et militants avec la "ligne dure " de la nécessité d'interdiction de l'hébergement touristique, sans nécessairement que ce soient des positions votées par les membres et avec lesquelles elles et ils sont d'accord. Notre propos ici n'est pas de discuter des enjeux sociaux, politiques et économiques liés à l'hébergement touristique ou encore du PSL privé, mais bien de donner des exemples concrets de situations dans lesquelles les travailleuses et travailleurs des comités logement défendent des positions qui viennent $\mathrm{du}$ " haut " et non pas de la base militante. 
À ce titre, il apparaît pertinent d'amorcer la réflexion par un questionnement plus global sur la nature des aspirations véhiculées par les travailleuses et travailleurs et les besoins des gens qui forment la base militante des groupes. Il y a trente ans déjà, Lamoureux, Mayer et Panet-Raymond (1984) relevaient des insatisfactions devant une certaine reproduction des rapports sociaux dominants au sein même des groupes communautaires. Par ailleurs, nous croyons qu'il convient de se questionner sur les idéaux démocratiques qui guident l'organisation de la contestation des locataires devant leurs conditions locatives. Découlent-ils d'une certaine vision romantique portée par des intellectuelles et intellectuels qui tendraient à glorifier le passé des groupes communautaires de défense des droits? Contribuent-ils à remettre en question des dynamiques et des pratiques d'intervention et de mobilisation qui fonctionnent en regard d'idéaux théoriques? Une mise en garde s'imposerait alors, car de tels parangons pourraient contribuer à susciter des effets pervers, c'est-à-dire des situations contraires aux objectifs recherchés, dont la désaffectation des militantes et militants.

\section{Notes}

1 Au cours des années 1970, plusieurs programmes d'emplois communautaires temporaires ont été mis en place, dont le Programme d'initiatives locales (PIL) et Canada au travail. Ces derniers avaient le double objectif de créer des emplois, notamment pour les chômeurs, et d'améliorer la situation des collectivités, notamment en favorisant la création de nouveaux organismes et services (Gyarmati, et collab., 2008).

2 Le Comité logement Centre Sud a changé son nom pour celui de Comité logement Ville-Marie en 2010.

3 Ce chiffre est tiré des sites Internet du RCLALQ et du FRAPRU consultés le 5 septembre 2014. Bien que ces listes puissent être incomplètes, nous vous invitons à les consulter à http://www. rclalq.qc.ca/index.php?z=21 et http://frapru.qc.ca/?-Liste-des-membres-

$4 \quad$ Voir la référence 2.

5 Le terme « logement social » réfere aux logements publics que sont les habitations à loyer modique (HLM) et aux logements communautaires que sont les coopératives d'habitation et les organismes sans but lucratif (OSBL) d'habitation. Le terme « logement privé » réfere, pour sa part, à tous les autres logements.

6 Une première reconnaissance officielle de cet apport s'est faite en 1991 avec l'adoption du projet de loi 120 (voir Trottier, 1991), puis une seconde en 1995 avec la création du Secrétariat à l'action communautaire autonome (SACA), qui vise à soutenir l'action communautaire et qui s'est accompagné de la création du Fonds d'aide à l'action communautaire autonome servant à financer l'action communautaire par le biais de trois programmes financiers : le programme de 
Soutien à la défense collective des droits, le programme de Soutien aux cibles prioritaires et le programme de Soutien au développement de l'action.

7 Centraide est une fondation publique dont la mission est de rassembler des ressources financières afin de financer des organismes communautaires travaillant sur différentes causes.

8 Très peu de recherches ont été menées sur le fonctionnement et les pratiques des comités logement au Québec. Les quelques auteures et auteurs qui abordent cette question sont Patenaude (1980), Boucher et Inkel (2001), Plamondon (2004), Charest (2004), Bencherki (2011), Auchinleck (2011), Breault (2015) et Goyer (2017).

9 Liste des dix-sept comités logement : Action Dignité de Saint-Léonard, Association des locataires de Villeray, Comité d'action Parc Extension, Comité logement Ahuntsic-Cartierville, Comité logement Ville-Marie, Comité logement de Lachine-Lasalle, Comité logement de la Petite Patrie, Comité logement de Montréal-Nord, Comité logement de Rosemont, Comité logement du Plateau Mont-Royal, Comité logement Saint-Laurent, Entraide Logement Hochelaga-Maisonneuve, Infologis de l'Est de l'Île de Montréal, OEIL Côtes-des-Neiges, POPIR Comité logement, Projet Genèse, R.I.L.

10 À l'exception d'un organisme qui a un mandat d'éducation populaire auprès des citoyennes et citoyens en ce qui a trait à l'aménagement urbain.

11 À l'exception de la répondante no 6, qui travaille toutefois en étroite collaboration avec une collègue dont le titre est celui d'organisatrice communautaire.

12 La Régie du logement est le tribunal qui, au Québec, a la compétence exclusive pour entendre toute demande relative au bail d'un logement ayant pour objet une réclamation inférieure à $80000 \$$.

13 Pour prendre connaissance de la mission de la Régie du logement, voir l'article 5 de la Loi sur la Régie du logement (L.R.Q., chapitre R-8.1, art. 5).

14 Comité logement Saint-Louis (1980).

15 Voir notamment http://www.rclalq.qc.ca/index.php?perma=1317843094

16 Voir notamment http://www.newswire.ca/en/story/1170345/-r-e-p-r-i-s-e-avis-aux-medias-regiedu-logement-mepris-du-logement-corrige-le-rclalq

17 Voir notamment http://www.lapresse.ca/le-nouvelliste/actualites/201002/10/01-948118-la-regiedu-lentement.php

18 Voir notamment http://www.newswire.ca/en/story/1169865/avis-aux-medias-regie-du-logementmepris-du-logement-corrige-le-rclalq? relation=org

19 Le programme Supplément au loyer (PSL) offre une aide financière permettant aux locataires de payer un loyer correspondant à $25 \%$ de leur revenu. Il peut être versé sur le marché privé ou 
encore dans des logements sociaux et communautaires (OSBL et coopératives d'habitation). Il est versé de facto aux locataires de HLM.

20 Réf. du 20 juin 2017, http://www.frapru.qc.ca/budgetleitao

21 Cette approche est aussi désignée par l'expression bottom-up process en opposition à l'approche top-down.

\section{Bibliographie}

AUCHINLECK, Jennifer (2011). Enforcing the Right to Healthy Housing: Recourse for Montreal Tenants Facing Health and Safety Problems at Home, [mémoire de maîtrise], Montréal, McGill University, $148 \mathrm{p}$.

BENCHERKI, Nicolas (2011). Avoir ou ne pas être. La constitution possessive de l'organisation, [thèse de doctorat], Université de Montréal et Sciences Po Paris, 245 p.

BOUCHER, Jacques L., et André INKEL (2001). « Mouvement communautaire et logement social : les cas Logemen'occupe et Mon Chez Nous ", Chaire de recherche en développement communautaire (CRDC), Série Pratiques économiques et sociales, 37 p.

BOURQUE, Denis, et collab. (2007). «L'organisation communautaire au Québec: Mise en perspective des principales approches stratégiques d'intervention ", dans Denis Bourque, et collab. (dirs.), L’organisation communautaire : fondements, approches et champs de pratique, Québec, Presses de l'Université du Québec, p. 3-19.

BREAULT, Geneviève (2015). «Recherche et luttes sociales : retour sur une expérience empirique sur le terrain de la défense de droits des locataires ", Aporia, 6(2), p. 19-26.

CHAREST, René (2004). «Le parcours d'une pratique d'éducation populaire : Entrevue avec Bernard Vallée de L’Autre Montréal », Nouvelles pratiques sociales, Vol. 17, No 1, p. 33-40.

COMEAU, Yvan (2007). "L'approche d'action sociale ou sociopolitique en organisation communautaire ", dans Denis Bourque, et collab. (dirs.), L'organisation communautaire : fondements, approches et champs de pratique, Québec, Presses de l'Université du Québec, p. 81-100.

COMEAU, Yvan (2012). "Les stratégies d'intervention sociopolitique favorables à l'action collective ", Reflets : revue d'intervention sociale et communautaire, Vol. 18, No 1, p. 61-90.

COMITÉ LOGEMENT SAINT-LOUIS (1980). « L'organisation de la lutte pour le droit au logement des locataires et petits propriétaires du quartier Saint-Louis ", dans Pierre Hamel, Jean-François Léonard et Robert Mayer (dirs.), Les mobilisations populaires urbaines, Montréal, Nouvelle optique, p. 91-97.

COMITÉ LOGEMENT SAINT-LOUIS (1979). Réponse au questionnaire d'enquête sur le fonctionnement. 
COMITÉ OPÉRATION-LOGEMENT HOCHELAGA-MAISONNEUVE (1979). Réponse au questionnaire d'enquête sur le fonctionnement.

DEPELTEAU, Julie (2013). Enjeux liés au financement et à la gouvernance des organismes communautaires québécois, [revue de la littérature], Institut de recherche et d'informations socioéconomiques.

DEPELTEAU, Julie, Francis FORTIER et Guillaume HÉBERT (2013). Les organismes communautaires au Québec : financement et évolution des pratiques, [rapport de recherche], Institut de recherche et d'informations socio-économiques.

DESLAURIERS, Jean-Pierre (2014). Les groupes communautaires : vers un changement de paradigme?, Québec, Presses de l'Université Laval.

DUVAL, Michelle, et collab. (2005). Les organismes communautaires au Québec : pratiques et enjeux, Montréal, Chenelière Éducation.

FONTAINE, Annie (2013). « La quête d'autonomie de l'action communautaire au Québec. Mission ou illusion collective? ", Le sociographe, 2013/5, Hors-série 6, p. 205-219.

GOUVERNEMENT DU QUÉBEC (1978). Livre blanc sur les relations entre locateurs et locataires, Québec.

GOUVERNEMENT DU QUÉBEC (2004). Cadre de référence en matière d'action communautaire, Québec.

GOYER, Renaud (2017). "Salubrité et santé dans l'action collective en matière de logement à Montréal », Lien social et Politiques, No 78, p. 70-88.

GUBERMAN, Nancy, et collab. (1994). «Des questions sur la culture organisationnelle des organismes communautaires ", Nouvelles pratiques sociales, Vol. 7, N 1, p. 45-62.

GYARMATI, David, et collab. (2008). Mobiliser les collectivités pour le développement local : L'évaluation des effets du Projet d'innovation en emploi communautaire sur les collectivités, Société de recherche sociale appliquée (SRSA).

L'ÉCUYER, Jean-Pierre (1987). "L'analyse de contenu : notions et étapes ", dans Jean-Pierre Deslauriers (dir.), Les méthodes de la recherche qualitative, Québec, Presses de l'Université du Québec, p. 49-65.

LAMOUREUX, Henri (2010). La pratique de l'action communautaire autonome, Québec, Presses de l'Université du Québec.

LAMOUREUX, Henri (1996). Le citoyen responsable : l'éthique de l'engagement social, Montréal, VLB éditeur.

LAMOUREUX, Henri, Robert MAYER et Jean PANET-RAYMOND (1984). L'intervention communautaire, Montréal, Éditions Saint-Martin. 
MATHIEU, Réjean, et Clément MERCIER (1991). "Assistés sociaux et sans-emploi, locataires, consommateurs... ", dans Laval Doucet et Louis Favreau (dirs.), Théorie et pratiques en organisation communautaire, Québec, Presses de l'Université du Québec, p. 351-376.

MAYER, Robert, et Jean PANET-RAYMOND (1991). "L'action communautaire de défense des droits sociaux ", dans Laval Doucet et Louis Favreau (dirs.), Théorie et pratiques en organisation communautaire, Québec, Presses de l'Université du Québec, p. 97-118.

MAYER, Robert, Henri LAMOUREUX et Jean PANET-RAYMOND (2008). "L'évolution des pratiques communautaires au Québec ", dans Henri Lamoureux, et collab. (dirs.), La pratique de l'action communautaire, Québec, Presses de l'Université du Québec, p. 11-97.

MILOT, Pierre (1979). Rapport de l'enquête du comité de direction auprès des groupes membres du Regroupement pour le gel des loyers, Regroupement pour le gel des loyers.

PATENAUDE, Jules (1980). "La mobilisation des locataires au Comité de logement Rosemont ", International Review of Community Development, No 4, p. 98-100.

PLAMONDON, Denis (2004). Loge m'entraide ou la solidarité des locataires au cœur du changement, Collection Les travaux du Collectif IDÉES — UQAC, Université du Québec à Chicoutimi.

RENÉ, Jean-François (2009). "L'individualisation de l'intervention dans les organismes communautaires : levier ou barrière à la prise en charge démocratique? ", Nouvelles pratiques sociales, Vol. 22, No 1, p. 111-124.

RENÉ, Jean-François, et Jean PANET-RAYMOND (1984). "Faut-il brûler les pancartes? Le mouvement populaire aujourd'hui », ACEF du Centre de Montréal et journal La Criée, Montréal.

SHRAGGE, Eric (2006). Action communautaire : dérives et possibles, Les Éditions Écosociété, Montréal.

TROTTIER, Sandra (1991). "La réforme Coté et les organismes communautaires ", Nouvelles pratiques sociales, Vol. 4, No 1, p. 147-152.

VILLE DE MONTRÉAL (2009). Profil statistique en habitation de la ville de Montréal, Service de la mise en valeur du territoire et du patrimoine, Direction de l'habitation de la Ville de Montréal, $35 \mathrm{p}$.

WYERS, Norman L. (1991). " Policy-Practice in Social Work: Models and Issues », Journal of Social Work Education, Vol. 27, No 3, p. 241-250. 